that the concussion will raise a cloud of finely divided solid matter from the bottom of the tube along which the flame will be propagated and be driven out at the other end. $A$ (see figure) is a wooden box 12 inches long, 8 broad, and 6 deep, closed on all sides, with the exception of a rectangular hole $\left(3 \frac{1}{4} \times 2 \frac{3}{4}\right.$ inches), into which can be inserted a long narrow rectangular tube (B), also of wood, which may be 20 feet or more in length ; the upper side $(c c)$ of this tube is binged, and along the bottom is strewed a thin layer of finely-divided dry coal-dust, or, what is better in the lecture-room, lycopodium powder. Into the wooden box, which in my apparatus has a cubic content of more than a gallon ( 5 litres) is placed about $1 \frac{3}{4}$ pints (say I litre) of coal-gas; this can be most readily effected by pouring this amount of water into the box and displacing it over the water-trough by a current of the coal-gas. The opening is then closed by a sliding lid, and the gaseous contents are mixed by violently shaking the box for a minute or so. The end of the long tube (along which the powder or dust has been strewn, and the lid $c c$ pushed down) is then inserted into the box, and the gaseous mixture is fired by thrusting a lighted taper through a small hole $(d)$ at the end just where the tube enters the box. The mixture of coal-gas and air explodes, and the flame rushes along the whole length of the tube with astonishing velocity, and is driven, often to a distance of six or seven feet, out at the other end, and is followed by a cloud of smoke.

The experiment is unaccompanied by danger, and is so simple that it may be readily performed in a lectureroom. I showed it some time since to a number of colliers and others engaged in coal-mining, and it seemed to bring home to them far more forcibly than possibly any amount of mere description would have done, the real character of the phenomenon.

T. E. THORPE

\section{THE PHOTOGRAPHIC SPECTRUM OF THE GREAT NEBULA IN ORION 1}

I AST evening (March 7 ) I succeeded in obtaining a photograph of the spectrum of the great nebula in Orion, extending from a little below $F$ to beyond $M$ in the ultra-violet.

The same spectroscope and special arrangements, attached to the 18 -inch Cassegrain telescope with metallic speculum belonging to the Royal Society, were employed which have been described in my paper on "The Photographic Spectra of Stars" (Phil. Trans., I88o, p. 672).

The exposure was limited by the coming up of clouds to forty-five minutes. The opening of the slit was made wider than during my work on the stars.

The photographic plate shows a spectrum of bright Jines, and also a narrower continuous spectrum which I think must be due to stellar light. The bright stars forming the trapezium in the "fish's mouth" of the nebula were kept close to the side of the slit, so that the light from the adjacent brightest part of the nebula might enter the slit.

Outside this stronger continuous spectrum I suspect an exceedingly faint trace of a continuous spectrum. In the diagram which accompanies this paper the spectrum of bright lines only is shown, which is certainly due to the light of the nebula.

In my papers on the visible spectrum of the nebula in Orion, and other nebulæ (Phil. Trans., 1864, p. 437, and I 868, p. 540; also Proc. Roy. Soc., 1865, p. 39, and I872, p. 380 ), I found four bright lines. The brightest line, wave-length 5005 , is coincident with the less refrangible component of the double line which is strongest in the spectrum of nitrogen. The second line has a wave-length of 4957 on Angström's scale. The other two lines are

I Paper read at the Royal Society, March I6, by William Huggins, coincident with two lines of hydrogen, $\mathrm{H} \beta$ or $\mathrm{F}$, and $\mathrm{H} \gamma$ near $G$.

In the photograph, these lines which had been observed in the visible spectrum are faint, but can be satisfactorily recognised and measured. In addition to these known lines, the photograph shows a relatively strong line in the ultra-violet, which has a wave-length 3730 , or nearly so. The wide slit does not permit of quite the same accuracy of determination of position as was possible in the case of the spectra of stars. For the same reason, I cannot be certain whether this new line is really single, or is double or multiple. In the diagram the line is represented broad, to indicate its relative great intensity.

This line appears to correspond to $\zeta$ of the typical spectrum of white stars (Phil. Trans., 1880, p. 677). In these stars the line is less strong than the hydrogen line near $G$; but in the nebula, it is much more intense than

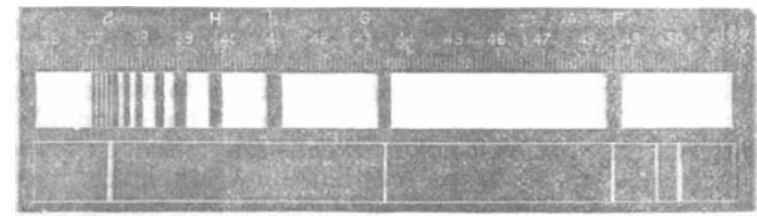

$\mathrm{H} \gamma$. In the nebula, the hydrogen lines $\mathrm{F}$ and $\mathrm{H} \gamma$ are thin and defined, while in the white stars they are broad, and winged at the edges. The typical spectrum has been added, for the sake of comparison, to the diagram.

I cannot say positively, that the lines of hydrogen between $\mathrm{H} \gamma$ and the line at 3730 are absent. If they exist in the spectrum of the nebula, they must be relatively very feeble. I suspect, indeed, some very faint lines at this part of the spectrum, and possibly beyond $\lambda 3730$, but I am not certain of their presence. I hope, by longer exposures and with more sensitive plates, to obtain information on this and other points. It is, perhaps, not too much to hope, that the further knowledge of the spectrum of the nebulæ afforded us by photography, may lead, by the help of terrestrial experiments, to more definite information as to the state of things existing in those bodies.

THE ACTION OF CARBONATE OF AMMONIA ON THE ROOTS OF CERTAIN PLANTS, AND ON CHLOROPHYLL BODIES ${ }^{1}$

\section{Roots}

THE observations which led to the first of these papers were originally made many years ago on Euphorbia peplus, and have now been extended to other genera. A plant of $E$. peplus having been dug up and carefully washed, the smaller rootlets may be placed under the microscope without further preparation, the thicker roots may be examined by means of sections. If such roots are left, before being examined, in a solution of carbonate of ammonia ( 1 to 7 per 1000) for a short time (varying from a few minutes to several hours), they present a wonderfully changed appearance. The most striking alteration is that the surface of the root assumes a long $i-$ tudinally striped appearance, due to longitudinal rows of darker brown cells, alternating with lighter coloured rows. The darker colour is seen under a high power to be due to the presence of innumerable rounded granules of a brown tint, which the lighter-coloured cells are without. Similar brown granules are deposited in cells scattered throughout the parenchyma, and markedly in the elongated endoderm cells surrounding the vascular bundle.

The granules are apparently neither resinows nor fatty, for they are not removed by alcohol or ether; they are,

Abstract by Mr. Francis Darwin of two papers by Mr. Charles Darwin read before the Linnean Society on March 16. 